\title{
Increasing Dose of Autologous Bone Marrow Mononuclear Cells Transplantation Is Related to Stroke Outcome: Results from a Pooled Analysis of Two Clinical Trials
}

\author{
Francisco Moniche, ${ }^{1,2}$ Paulo Henrique Rosado-de-Castro, ${ }^{3}$ \\ Irene Escudero, ${ }^{1,2}$ Elena Zapata, ${ }^{1,2}$ Francisco Javier de la Torre Laviana, ${ }^{1,2}$ \\ Rosalia Mendez-Otero, ${ }^{4}$ Magdalena Carmona, ${ }^{5}$ Pilar Piñero, ${ }^{6}$ \\ Alejandro Bustamante, ${ }^{7}$ Lucía Lebrato, ${ }^{1}$ Juan Antonio Cabezas, ${ }^{1}$ Alejandro Gonzalez, ${ }^{6}$ \\ Grabriel R. de Freitas, ${ }^{8}$ and Joan Montaner ${ }^{2,7}$ \\ ${ }^{1}$ Department of Neurology, Hospital Universitario Virgen del Rocío, 41013 Seville, Spain \\ ${ }^{2}$ Instituto de Biomedicina de Sevilla-IBiS, Hospital Universitario Virgen del Rocío, 41013 Seville, Spain \\ ${ }^{3}$ Instituto de Ciências Biomédicas, Federal University of Rio de Janeiro, 21044-020 Rio de Janeiro, RJ, Brazil \\ ${ }^{4}$ Instituto de Biofísica Carlos Chagas Filho, Federal University of Rio de Janeiro, 21044-020 Rio de Janeiro, RJ, Brazil \\ ${ }^{5}$ Department of Hematology, Hospital Universitario Virgen del Rocío, 41013 Seville, Spain \\ ${ }^{6}$ Department of Radiology, Hospital Universitario Virgen del Rocío, 41013 Seville, Spain \\ ${ }^{7}$ Neurovascular Research Laboratory, Institut de Recerca Vall d'Hebron, Hospital Vall d'Hebron, 08035 Barcelona, Spain \\ ${ }^{8}$ D'Or Institute for Research and Education, 22281-100 Rio de Janeiro, RJ, Brazil
}

Correspondence should be addressed to Francisco Moniche; pmoniche@gmail.com

Received 5 February 2016; Revised 21 June 2016; Accepted 28 June 2016

Academic Editor: Renke Li

\begin{abstract}
Copyright (C) 2016 Francisco Moniche et al. This is an open access article distributed under the Creative Commons Attribution License, which permits unrestricted use, distribution, and reproduction in any medium, provided the original work is properly cited.

Background and Purpose. BM-MNC transplantation improves recovery in experimental models of ischemic stroke. Clinical trials are ongoing to test efficacy in stroke patients. However, whether cell dose is related to outcomes is not known. Methods. We performed a pooling data analysis of two pilot clinical trials with autologous BM-MNCs transplantation in ischemic stroke patients. Cell dose and route were analyzed to evaluate their relation to good outcome (m-Rankin scale [mRS] score 0-2) at 6 months. Results. Twentytwo patients were included. A median of $153 \times 10^{6}\left( \pm 121 \times 10^{6}\right)$ BM-MNCs was injected. Intra-arterial route was used in $77.3 \%$ of cases. A higher number of cells injected were associated with better outcomes at 180 days $\left(390 \times 10^{6}[320-422]\right.$ BM-MNCs injected in those patients with $\mathrm{mRS}$ of $0-2$ at 6 months versus $130 \times 10^{6}$ [89-210] in those patients with mRS 3-6, $\left.p=0.015\right)$. In the intraarterially treated patients, a strong correlation between dose of cells and disability was found $(r=-0.63, p=0.006)$. A cut point of $310 \times 10^{6}$ injected cells predicted good outcome with $80 \%$ sensitivity and $88.2 \%$ specificity. Conclusions. Similar to preclinical studies, a higher dose of autologous BM-MNC was related to better outcome in stroke patients, especially when more than $310 \times$ $10^{6}$ cells are injected. Further interventional studies are warranted to confirm these data.
\end{abstract}

\section{Introduction}

Stroke is one of the leading causes of morbidity and longterm disability in the world, with about one-third of survivors being permanently disabled [1]. In the very acute phase of stroke thrombolytics and endovascular thrombectomy can reduce stroke disability; however, there are few options for recovery once the neurological deficits are established. In recent years, extensive cell therapy preclinical research has demonstrated a neurorestorative effect after cerebral 
ischemia, improving neurological outcomes even in the long term [2-4]. Amongst the most promising are bone marrow mononuclear cells (BM-MNCs), which have consistently demonstrated efficacy in animal stroke models in different laboratories and species [4-6]. These cells have the advantage of being rapidly isolated from bone marrow, do not require culture, and can be injected within hours from bone marrow aspiration and therefore suited for autologous administration even in the acute phase of stroke. In the last years, some preliminary phase I/II trials have shown the safety and feasibility of autologous bone marrow transplantation in stroke patients [7-11]. However, many questions regarding dose, route, and type of cells need to be addressed before starting phase III trials.

A wide range of cells numbers has been used for transplantation in animal stroke models and in clinical trials. While in preclinical studies there is strong evidence that a higher dose of cells increases the probability of a good neurological outcome $[12,13]$ the optimal number of cells to be transplanted for ischemic stroke is largely unknown. This raises the question of whether a higher dose of BMMNCs will produce a greater effect in recovery in stroke patients.

In order to evaluate the relation of dose and recovery of neurological deficit in stroke patients treated with BMMNCs, we performed a pooling data of clinical trials with autologous BM-MNCs transplantation.

\section{Material and Methods}

2.1. Pooled Clinical Trials. We combined individual data from two different pilot phase I/II clinical trials, which were designed to assess the safety and feasibility of BM-MNCs transplantation in ischemic stroke patients and conducted in Spain and Brazil [7-9]. Thirty-two patients were included in both clinical trials. Of them, ten patients were controls and twenty-two patients were actively treated with BMMNCs. Patients included had an ischemic stroke in the MCA territory. Inclusion and exclusion criteria were largely similar between the two trials. Main differences included different time windows, with patients treated in the Spanish trial within 5-9 days of stroke onset and those treated in the Brazilian trial within 90 days from stroke onset; National Institute of Health stroke scale (NIHSS) score was $\geq 8$ at inclusion in the Spanish trial and 4 to 20 in the Brazilian trial; and age inclusion criteria had the upper limit in 80 years in the Spanish trial and 75 years in the Brazilian trial.

In both trials patients with lacunar or hemorrhagic stroke were excluded. Other exclusion criteria were pregnancy, history of neoplasia, life threatening illness, hematological diseases, significant previous disability (prestroke modified Rankin Scale [mRS] score $\geq 3$ ), and severe comorbidity (severe hepatic or renal dysfunction) that would preclude follow-up.

Treatment and outcome assessment were done according to the individual study protocols that were approved by the relevant institutional review boards. Written informed consent was obtained from each patient or their representatives. The trials were registered with clinicaltrials.gov (trial identification numbers NCT00761982 [7] and NCT00473057 $[8,9])$.

2.2. Cell Therapy Procedures. Transplantation procedure was done in the BM-MNC-treated group as previously described $[7,8]$. In brief, 50-80 milliliters of bone marrow was obtained by puncture in the posterior iliac crest. The aspirate was centrifuged on a Ficoll density gradient to isolate the mononuclear cells, which were injected in the M1 segment of the infarct-related MCA in approximately 10 minutes. In five patients in the Brazilian trial, BM-MNCs were administered intravenously into the antecubital vein. In every patient, the injection was performed approximately at the rate of $1 \mathrm{~mL} / \mathrm{min}$. No bone marrow aspiration or sham injection was performed in the control group.

2.3. Outcomes Evaluation. Clinical and functional evaluation ( $\mathrm{m}$-Rankin Scale and NIHSS) were performed after transplantation and 1,3 , and 6 months after the stroke.

In the pooled analysis, the primary outcome measure was the score on the mRS at 6 months dichotomized between good outcome ( $\mathrm{mRS} 0$ to 2 ) and unfavourable outcome (mRS 3 to 6$)$.

Modified Rankin Scale score measures functional outcome after stroke [21]. Scores range from 0 to 6: 0 indicating no symptoms at all; 1 indicating no significant disability despite symptoms, being able to carry out all usual duties and activities; 2 indicating slight disability, being unable to carry out all previous activities but able to look after own affairs without assistance; 3 indicating moderate disability, requiring some help, but being able to walk without assistance; 4 indicating moderately severe disability, being unable to walk without assistance and unable to attend to own bodily needs without assistance; 5 indicating severe disability, being bedridden, incontinent, and requiring constant nursing care and attention; and 6 indicating death.

2.4. Statistical Analysis. The Kolmogorov-Smirnov test was applied to verify if the variables followed a normal distribution. When the variables were not normally distributed, comparisons between groups were made using the MannWhitney $U$ tests to detect differences in the distribution of samples and Spearman's Rho coefficient to assess the relationship between two quantitative variables. Categorical data are expressed as percentages and analyzed using the Chi-square test $\left(\chi^{2}\right)$ or Fisher's exact test where appropriate. Spearman's rank correlation coefficient was used to assess the association between outcomes (mRS and NIHSS) and the number of BM-MNCs injected. The number of BM-MNCs injected was dichotomized at the best cut-off point for a better accuracy in predicting good outcome at six months using receiver operating characteristics (ROC) curves. All statistical analyses were performed using the SPSS software package version 18.0 for Windows (SPSS Inc., Chicago, IL, USA). Differences were considered to be statistically significant when two-tailed $p$ values were less than 0.05 . 


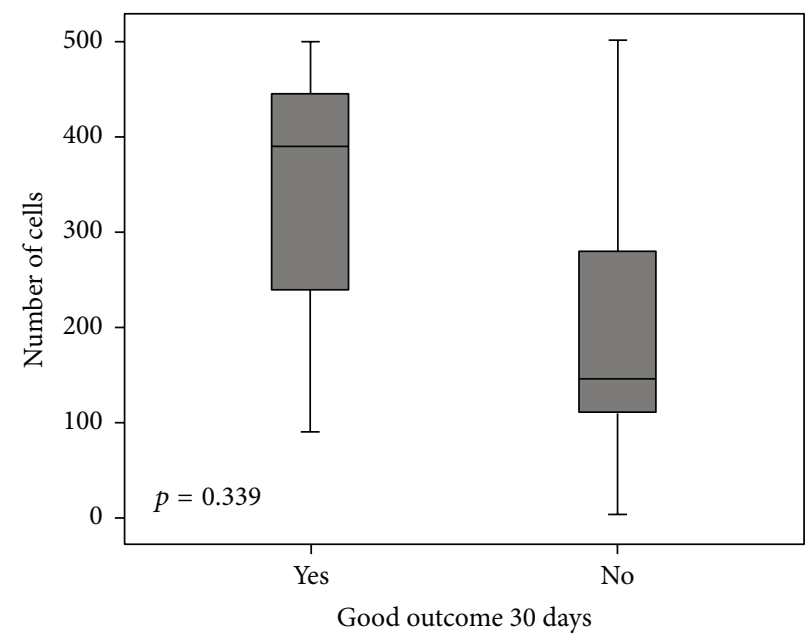

(a)

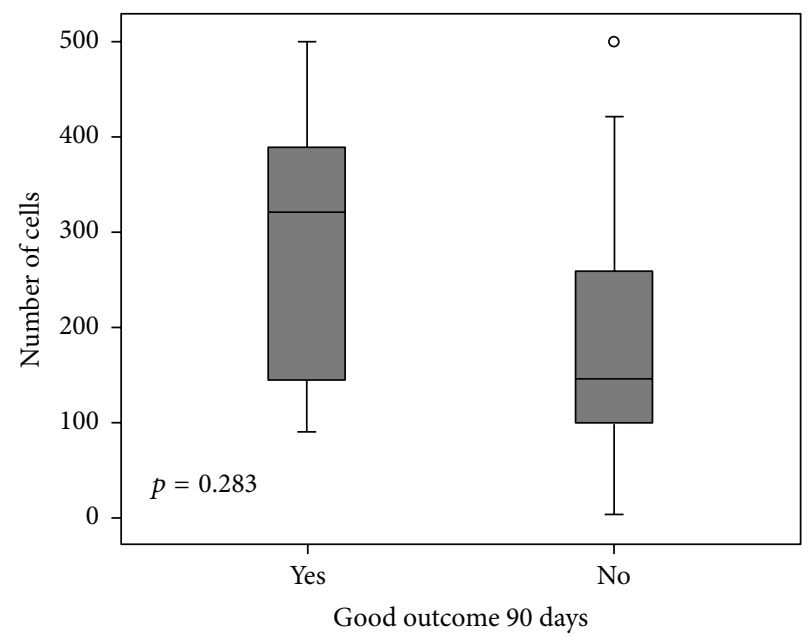

(b)

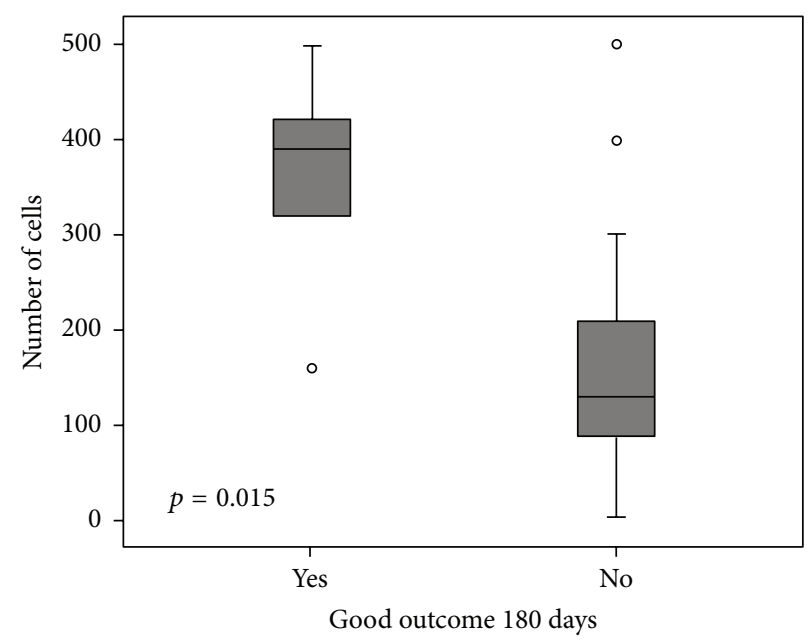

(c)

FIGURE 1: Box plot of the relation between number of injected cells and stroke outcomes during follow-up. Patients with good outcomes had no or mild disability (mRS of 0-2). Values are expressed in millions of cells. Small circles mean outliers.

\section{Results}

Of the 22 patients treated with BM-MNCs, $68.2 \%$ were men and mean age was 60.4 years $( \pm 14)$. Main risk factors were hypertension $(68.2 \%)$, diabetes (40.9\%), and dyslipidemia (40.9\%). All have a moderate-to-severe MCA stroke at inclusion with a median NIHSS score of 13.0 (IQR 9.75-16.9). Cardioembolic stroke was the most frequent mechanism of stroke (40.9\%) and intravenous thrombolysis was performed in the acute phase of stroke in $31.8 \%$ of patients (Table 1 ).

Both cohorts were very similar in baseline characteristics except for age (67.4 years of age in those patients included in the Spanish trial versus 54.6 in the Brazilian trial, $p=0.03$ ) and days from stroke to treatment (BM-MNCs injection at 6.3 days from stroke onset in the Spanish trial versus 58 days in the Brazilian trial, $p<0.001)$.

A median of $153 \times 10^{6}\left( \pm 121 \times 10^{6}\right)$ BM-MNCs was injected in the 22 cases. Intra-arterial route was used in 17 patients (77.3\% of cases).
Regarding safety, during follow-up there was no deaths, tumor formation, or stroke recurrence. Seven patients $(31.8 \%)$ had a partial seizure during follow-up and 4 patients (18.2\%) had a systemic infection (Table 2).

When evaluating dose of BM-MNCs administered, the higher number of injected cells was associated with better outcomes at 180 days $\left(390 \times 10^{6}\right.$ [320-422] BM-MNCs injected in those patients with mRS of $0-2$ versus $130 \times 10^{6}$ [89-210] in those patients with mRS 3-6 at 6 months, $p=$ 0.015) (Figure 1).

There were no significant correlations between number of cells and NIHSS or mRS at any time point when analyzing all the BM-MNC-treated patients included in the study $(n=$ 22 ), but there was a trend towards less disability when a higher number of cells were injected (mRS at 90 days $(r=$ $-0.372, p=0.088)$ and $\mathrm{mRS}$ at 180 days $(r=-0.389$, $p=0.074)$ ). However, when analyzing only those patients treated intra-arterially with BM-MNCs $(n=17)$, a strong negative correlation between dose of cells and $\mathrm{mRS}$ score at 
TABLE 1: Baseline characteristics of patient treated in both clinical trials.

\begin{tabular}{|c|c|c|c|c|}
\hline & All $(N=22)$ & Spanish trial $(N=10)$ & Brazilian trial $(N=12)$ & $p$ \\
\hline Age & $60.4 \pm 14$ & $67.4 \pm 13$ & $54.6 \pm 13$ & $0.03^{*}$ \\
\hline Gender (male) & $15(68.2 \%)$ & $5(50 \%)$ & $10(83.3 \%)$ & 0.17 \\
\hline Hypertension & $15(68.2 \%)$ & $6(60 \%)$ & $9(75 \%)$ & 0.62 \\
\hline Diabetes & $9(40.9 \%)$ & $3(30 \%)$ & $6(50 \%)$ & 0.41 \\
\hline Dyslipidemia & $9(40.9 \%)$ & $4(40 \%)$ & $4(33.3 \%)$ & 0.99 \\
\hline Tobacco & $5(22.7 \%)$ & $1(10 \%)$ & $4(33.3 \%)$ & 0.32 \\
\hline Cardiopathy & $2(9.1 \%)$ & $0(0 \%)$ & $2(16.7 \%)$ & 0.48 \\
\hline $\mathrm{AF}$ & $4(18.2 \%)$ & $2(20 \%)$ & $2(16.7 \%)$ & 0.99 \\
\hline \multicolumn{5}{|l|}{ TOAST } \\
\hline LAA & $3(13.6 \%)$ & $0(0 \%)$ & $3(25 \%)$ & \\
\hline $\mathrm{CE}$ & $9(40.9 \%)$ & $4(40 \%)$ & $5(41.7 \%)$ & \\
\hline LAC & $1(4.5 \%)$ & $1(10 \%)$ & $0(0 \%)$ & 0.39 \\
\hline UND & $7(31.8 \%)$ & $4(40 \%)$ & $3(25 \%)$ & \\
\hline OTH & $2(9.1 \%)$ & $1(10 \%)$ & $1(8.3 \%)$ & \\
\hline \multicolumn{5}{|l|}{ Side } \\
\hline Right & $9(40.9 \%)$ & $4(40 \%)$ & $5(41.7 \%)$ & \\
\hline Left & $13(59.1 \%)$ & $6(60 \%)$ & $7(58.3 \%)$ & 0.99 \\
\hline VB & - & - & - & \\
\hline IV thrombolysis & $7(31.8 \%)$ & $4(40 \%)$ & $3(25 \%)$ & 0.65 \\
\hline IA therapy & $1(4.5 \%)$ & $0(0 \%)$ & $1(8.3 \%)$ & 0.99 \\
\hline NIHSS (baseline) & $13.0[9.7-16.0]$ & $15.5[10.7-18.0]$ & $11.5[9.0-14.5]$ & 0.07 \\
\hline Infarct volume $e^{\#}$ & $84.4 \pm 65.5$ & $62.0 \pm 60.5$ & $99.4 \pm 66.9$ & 0.22 \\
\hline Injection days & $34.5 \pm 32.4$ & $6.3 \pm 1.3$ & $58 \pm 26$ & $<0.001^{*}$ \\
\hline Number of cells & $153.5(100-320)$ & $138.5(76-210)$ & $223.5(128-395)$ & 0.20 \\
\hline
\end{tabular}

Values are expressed as means \pm SD. ${ }^{*} p<0.05 ;{ }^{\sharp} n=20$. BM-MNC indicates bone marrow mononuclear cell.

TABLE 2: Follow-up of patients treated with BM-MNCs.

\begin{tabular}{|c|c|c|c|c|}
\hline & All $(N=22)$ & Spanish trial $(N=10)$ & Brazilian trial $(N=12)$ & $p$ \\
\hline Death & - & - & - & - \\
\hline Stroke & - & - & - & - \\
\hline MI & - & - & - & - \\
\hline Infection & $4(18.2 \%)$ & $4(40 \%)$ & $0(0 \%)$ & $0.03^{*}$ \\
\hline $\mathrm{SICH}$ & - & - & - & - \\
\hline Allergic reaction & - & - & - & - \\
\hline Tumors & - & - & - & - \\
\hline Seizure & $7(31.8 \%)$ & $2(20 \%)$ & $5(41.7 \%)$ & 0.38 \\
\hline NIHSS 30 & $9.0[6.0-12.0]$ & $9.0[7.0-13.0]$ & $10.0[5.2-12.0]$ & 0.80 \\
\hline NIHSS 90 & $6.5[4.7-11.2]$ & $6.5[5.7-11.7]$ & $7.5[4.0-11.7]$ & 0.77 \\
\hline NIHSS 180 & $6.0[4.0-10.2]$ & $6.0[3.7-11.2]$ & $8.0[4.2-10.7]$ & 0.67 \\
\hline$m R S \leq 230$ & $3(13.6 \%)$ & $1(10 \%)$ & $2(16.7 \%)$ & 0.99 \\
\hline $\mathrm{mRS} \leq 290$ & $5(22.7 \%)$ & $1(10 \%)$ & $4(33.3 \%)$ & 0.32 \\
\hline $\mathrm{mRS} \leq 2180$ & $5(22.7 \%)$ & $2(20 \%)$ & $3(25 \%)$ & 0.99 \\
\hline
\end{tabular}

${ }^{*} p<0.05$.

6 months was found $(r=-0.63, p=0.006)$. Also, there was a significant relationship between dose of intra-arterial BM-MNCs and disability in follow-up $\left(390 \times 10^{6}[240-461]\right.$ BM-MNCs injected in those patients with mRS of $0-2$ at 6 months versus $121 \times 10^{6}$ [79-208] in those patients with mRS 3-6, $p=0.009$ ). The low number of patients treated intravenously ( $n=5$ ) did not allow a separate analysis of the relationship between number of cells injected and outcomes in this subgroup of patients.

When analyzing receiver operating characteristics (ROC) curves, a cut point of $310 \times 10^{6}$ cells injected predicted a good outcome, with no or mild disability at 180 days after stroke 
( $\mathrm{mRS}$ of $0-2$ ) with a sensitivity of $80 \%$ and specificity of $88.2 \%$ (Figure 2).

\section{Discussion}

This pooled analysis of two different clinical trials with autologous BM-MNCs injection in ischemic stroke patients gives some light about optimal doses of cells to be tested in clinical trials, especially when intra-arterial route is used. To the best of our knowledge, we describe for the first time a significant relationship between higher dose of BM-MNCs and better outcomes in stroke patients and a possible efficacy dose cut-off for cell therapy trials.

This relationship is plausible as in preclinical studies there is strong evidence of the importance of cell dose in neurological outcomes after brain ischemia. In a recent meta-analysis of preclinical studies with mesenchymal stem cells (MSCs) for cerebral ischemia, authors found a negative correlation between dose of cells and neurological deficit during follow-up $(r=-0.63, p<0.001)$ [13], similar to our findings.

Since preclinical data indicate that dose is an important factor in optimizing cell therapy, low doses might not improve functional outcomes and larger number of cells might be unnecessary [22]. Our data indicates that the optimal threshold of transplanted cells is probably around $310 \times 10^{6} \mathrm{BM}$ MNCs in order to obtain good functional outcome with high probability amongst treated stroke patients.

However, available data about dose of cells in humans is conflicting. In line with our results, Taguchi et al. evaluated in a clinical trial two different doses of BM-MNCs administered intravenously in stroke patients after 7-10 days of stroke onset $\left(250 \times 10^{6}\right.$ and $340 \times 10^{6}$ cells in the lower and higher dose groups, resp.) and although it was a phase I/IIa clinical trial not designed to test efficacy, authors described a trend towards improved neurological outcomes in those patients receiving the higher dose of bone marrow cells [20].

On the other side, Prasad et al. published a phase II trial including 120 stroke patients with fifty-eight of them being treated with intravenous injection of BM-MNCs, showing no relationship between cell dose and outcomes [10].

Also, in a meta-analysis of cell-based therapies for treating stroke patients [23], authors found that stem cell therapy was more effective with higher dose of cells and also when intra-arterial route was used. In Table 3, published clinical trials with BM-MNCs in stroke patients are listed. Unfortunately, we were not able to obtain the information of other published BM-MNCs clinical trials and include them in the pooled analysis. Although the relevance of cell dose in clinical trials with stroke patients is not clear, regarding safety, there is no data in the literature about potential negative outcomes when higher BM-MNCs doses are injected. However, future trials testing different doses of cells should evaluate carefully not only efficacy but also the safety of higher doses of cells.

Regarding the route, most of the patients included in this pooling data study were treated using intra-arterial route. We found a trend towards less disability when higher number of cells was injected when all the patients included were

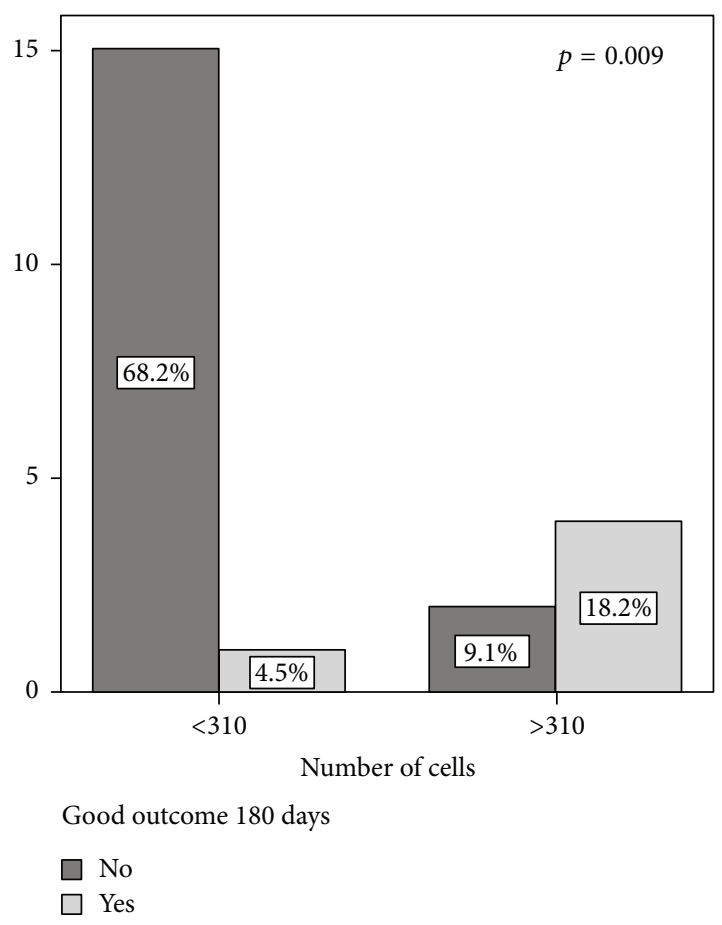

FIgURE 2: Analysis of a dose cut-off $\left(310 \times 10^{6} \mathrm{BM}-\mathrm{MNC}\right.$ injected $)$ and outcomes after 180 days. Patients with good outcomes had no or mild disability (mRS of $0-2$ ). Values are expressed in millions of cells.

analyzed. However, we found a strong negative correlation between cell dose and disability when intravenous patients were excluded from analysis, pointing to the hypothesis that the combination of higher number of cells and intra-arterial route could be a key factor to improve neurological outcomes in stroke patients.

Based on animal models of stroke it is not clear which route of delivery is preferable [13]. Intravenous cell delivery is a less invasive route and is increasingly used in clinical trials. However, preclinical studies have indicated that the intravenous injection leads to significant cell trapping in organs such as the lungs, liver, and spleen, with a small number of cells reaching the ischemic brain $[12,24]$. Previous observations showed that delivery routes dramatically affect the migration and distribution of grafted cells and that administration of bone marrow-derived cells using more invasive methods such as intra-arterial route may provide significantly greater benefit in stroke $[13,24]$. Despite the concern for the possibility of cerebral microembolism during intra-arterial injection leading to new focal ischemic lesions and worsening of neurological deficit, we did not detect new strokes or neurological deterioration after intra-arterial injection of BM-MNCs. Also, in preclinical studies it has been stated that vascular occlusion is related to the type of cells transplanted [25]. Although intra-arterial delivery of MSCs increased the risk of new ischemic lesions and mortality in rats [26], the intra-arterial injection of BM-MNCs was safe and did not reduce cerebral perfusion $[5,25,27]$. This may be 


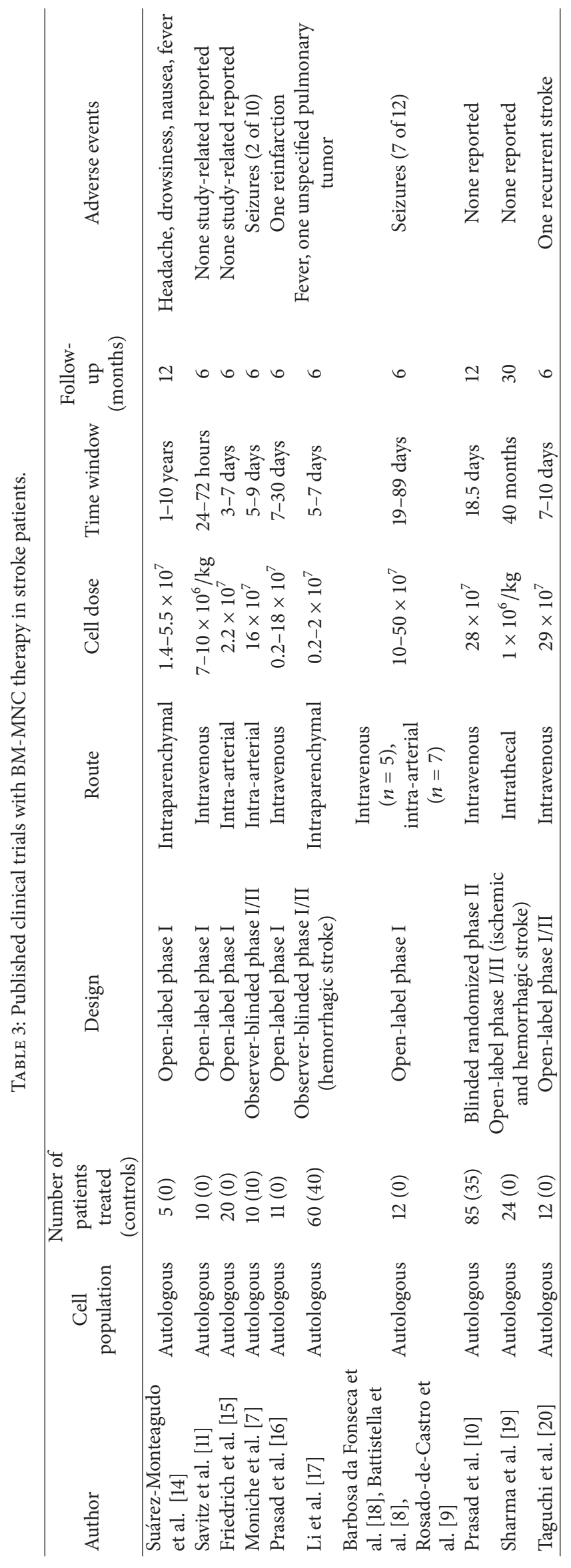


related to the smaller size of BM-MNCs compared to MSCs [25].

Although the mechanisms underlying cell therapy recovery are unclear, a potential explanation of the relevance of dose could be the secretion of cytokines and growth factors by BM-MNCs [25]. These cytokines are involved in angiogenesis and neurogenesis but also can reduce proinflammatory response after stroke $[5,6]$. In this line, our group described previously a significant negative correlation between number of cells injected and several cytokines such as MMP-2, playing a possible anti-inflammatory role and a positive correlation with GM-CSF and PDGF-BB, factors related to brain plasticity [28]. Therefore, we could hypothesize that higher number of cells transplanted could have greater neurorestorative effects in stroke patients through secretion of larger amount of some beneficial cytokines.

The main limitation of this study is that, due to the small sample size, logistic regression analysis could not be performed to evaluate the independent predictors of good outcome. However, our results are similar to the strong evidence previously described in stroke animal models and the meta-analysis of published clinical trials [23]. To confirm the relationship between cell dose and outcomes, a dosefinding multicenter clinical trial is ongoing in ischemic stroke patients treated with different doses of intra-arterial BMMNCs [29].

\section{Conclusions}

In conclusion, higher dose of autologous BM-MNC seems to be related to less disability in ischemic stroke patients, similar to preclinical studies, especially when more than $310 \times 10^{6}$ cells are injected. This relationship seems to be stronger when injection is performed intra-arterially. Further interventional studies are warranted to confirm these data.

\section{Competing Interests}

The authors declare that they have no competing interests.

\section{Acknowledgments}

This study is funded by grants from Junta de Andalucía [TCRM 0001/2006], Fundación Mutua Madrileña, and Instituto de Salud Carlos III [PI15/01197].

\section{References}

[1] A. S. Go, D. Mozaffarian, V. L. Roger et al., "Heart disease and stroke statistics-2013 update: a report from the American Heart Association," Circulation, vol. 127, no. 1, pp. e6-e245, 2013.

[2] M. Bacigaluppi, S. Pluchino, L. Peruzzotti-Jametti et al., "Delayed post-ischaemic neuroprotection following systemic neural stem cell transplantation involves multiple mechanisms," Brain, vol. 132, no. 8, pp. 2239-2251, 2009.

[3] S. I. Savitz, "Cell therapies: careful translation from animals to patients," Stroke, vol. 44, supplement 1, pp. S107-S109, 2013.
[4] Z. G. Zhang and M. Chopp, "Neurorestorative therapies for stroke: underlying mechanisms and translation to the clinic," The Lancet Neurology, vol. 8, no. 5, pp. 491-500, 2009.

[5] M. Brenneman, S. Sharma, M. Harting et al., "Autologous bone marrow mononuclear cells enhance recovery after acute ischemic stroke in young and middle-aged rats," Journal of Cerebral Blood Flow \& Metabolism, vol. 30, no. 1, pp. 140-149, 2010.

[6] Y. Fujita, M. Ihara, T. Ushiki et al., "Early protective effect of bone marrow mononuclear cells against ischemic white matter damage through augmentation of cerebral blood flow," Stroke, vol. 41, no. 12, pp. 2938-2943, 2010.

[7] F. Moniche, A. Gonzalez, J.-R. Gonzalez-Marcos et al., "Intraarterial bone marrow mononuclear cells in ischemic stroke: a pilot clinical trial," Stroke, vol. 43, no. 8, pp. 2242-2244, 2012.

[8] V. Battistella, G. R. de Freitas, L. M. B. da Fonseca et al., "Safety of autologous bone marrow mononuclear cell transplantation in patients with nonacute ischemic stroke," Regenerative Medicine, vol. 6, no. 1, pp. 45-52, 2011.

[9] P. H. Rosado-de-Castro, F. D. R. Schmidt, V. Battistella et al., "Biodistribution of bone marrow mononuclear cells after intra-arterial or intravenous transplantation in subacute stroke patients," Regenerative Medicine, vol. 8, no. 2, pp. 145-155, 2013.

[10] K. Prasad, A. Sharma, A. Garg et al., "Intravenous autologous bone marrow mononuclear stem cell therapy for ischemic stroke: a multicentric, randomized trial," Stroke, vol. 45, no. 12, pp. 3618-3624, 2014.

[11] S. I. Savitz, V. Misra, M. Kasam et al., "Intravenous autologous bone marrow mononuclear cells for ischemic stroke," Annals of Neurology, vol. 70, no. 1, pp. 59-69, 2011.

[12] B. Yang, R. Strong, S. Sharma et al., "Therapeutic time window and dose response of autologous bone marrow mononuclear cells for ischemic stroke," Journal of Neuroscience Research, vol. 89, no. 6, pp. 833-839, 2011.

[13] Q. Vu, K. Xie, M. Eckert, W. Zhao, and S. C. Cramer, "Metaanalysis of preclinical studies of mesenchymal stromal cells for ischemic stroke," Neurology, vol. 82, no. 14, pp. 1277-1286, 2014.

[14] C. Suárez-Monteagudo, P. Hernández-Ramírez, L. ÁlvarezGonzález et al., "Autologous bone marrow stem cell neurotransplantation in stroke patients. An Open Study," Restorative Neurology and Neuroscience, vol. 27, no. 3, pp. 151-161, 2009.

[15] M. A. G. Friedrich, M. P. Martins, M. D. Araújo et al., "Intraarterial infusion of autologous bone marrow mononuclear cells in patients with moderate to severe middle cerebral artery acute ischemic stroke," Cell Transplantation, vol. 21, supplement 1, pp. S13-S21, 2012.

[16] K. Prasad, S. Mohanty, R. Bhatia et al., "Autologous intravenous bone marrow mononuclear cell therapy for patients with subacute ischaemic stroke: a pilot study," Indian Journal of Medical Research, vol. 136, no. 2, pp. 221-228, 2012.

[17] Z.-M. Li, Z.-T. Zhang, C.-J. Guo, F.-Y. Geng, F. Qiang, and L.$\mathrm{X}$. Wang, "Autologous bone marrow mononuclear cell implantation for intracerebral hemorrhage-a prospective clinical observation," Clinical Neurology and Neurosurgery, vol. 115, no. 1, pp. 72-76, 2013.

[18] L. M. Barbosa da Fonseca, B. Gutfilen, P. H. Rosado de Castro et al., "Migration and homing of bone-marrow mononuclear cells in chronic ischemic stroke after intra-arterial injection," Experimental Neurology, vol. 221, no. 1, pp. 122-128, 2010.

[19] A. Sharma, H. Sane, N. Gokulchandran et al., "Autologous bone marrow mononuclear cells intrathecal transplantation in 
chronic stroke," Stroke Research and Treatment, vol. 2014, Article ID 234095, 9 pages, 2014.

[20] A. Taguchi, C. Sakai, T. Soma et al., "Intravenous autologous bone marrow mononuclear cell transplantation for stroke: phase1/2a clinical trial in a homogeneous group of stroke patients," Stem Cells and Development, vol. 24, no. 19, pp. 22072218, 2015.

[21] J. C. van Swieten, P. J. Koudstaal, M. C. Visser, H. Schouten, and J. van Gijn, "Interobserver agreement for the assessment of handicap in stroke patients," Stroke, vol. 19, no. 5, pp. 604-607, 1988.

[22] L.-Q. Wang, Z.-Z. Lin, H.-X. Zhang et al., "Timing and dose regimens of marrow mesenchymal stem cell transplantation affect the outcomes and neuroinflammatory response after ischemic stroke," CNS Neuroscience and Therapeutics, vol. 20, no. 4, pp. 317-326, 2014.

[23] H. Jeong, H. W. Yim, Y.-S. Cho et al., "Efficacy and safety of stem cell therapies for patients with stroke: a systematic review and single arm meta-analysis," International Journal of Stem Cells, vol. 7, no. 2, pp. 63-69, 2014.

[24] N. Kamiya, M. Ueda, H. Igarashi et al., "Intra-arterial transplantation of bone marrow mononuclear cells immediately after reperfusion decreases brain injury after focal ischemia in rats," Life Sciences, vol. 83, no. 11-12, pp. 433-437, 2008.

[25] J. Boltze, A. Arnold, P. Walczak, J. Jolkkonen, L. Cui, and D.-C. Wagner, "The dark side of the force-constraints and complications of cell therapies for stroke," Frontiers in Neurology, vol. 6, article 155, 2015.

[26] P. Walczak, J. Zhang, A. A. Gilad et al., "Dual-modality monitoring of targeted intraarterial delivery of mesenchymal stem cells after transient ischemia," Stroke, vol. 39, no. 5, pp. 1569-1574, 2008.

[27] A. Vasconcelos-dos-Santos, P. H. Rosado-de-Castro, S. A. Lopes de Souza et al., "Intravenous and intra-arterial administration of bone marrow mononuclear cells after focal cerebral ischemia: is there a difference in biodistribution and efficacy?" Stem Cell Research, vol. 9, no. 1, pp. 1-8, 2012.

[28] F. Moniche, J. Montaner, J. R. Gonzalez-Marcos et al., "Intraarterial bone marrow mononuclear cell transplantation correlates with GM-CSF, PDGF-BB, and MMP-2 serum levels in stroke patients: results from a clinical trial," Cell Transplantation, vol. 23, supplement 1, pp. S57-S64, 2014.

[29] F. Moniche, I. Escudero, E. Zapata-Arriaza et al., "Intra-arterial bone marrow mononuclear cells (BM-MNCs) transplantation in acute ischemic stroke (IBIS trial): protocol of a phase II, randomized, dose-finding, controlled multicenter trial," International Journal of Stroke, vol. 10, no. 7, pp. 1149-1152, 2015. 

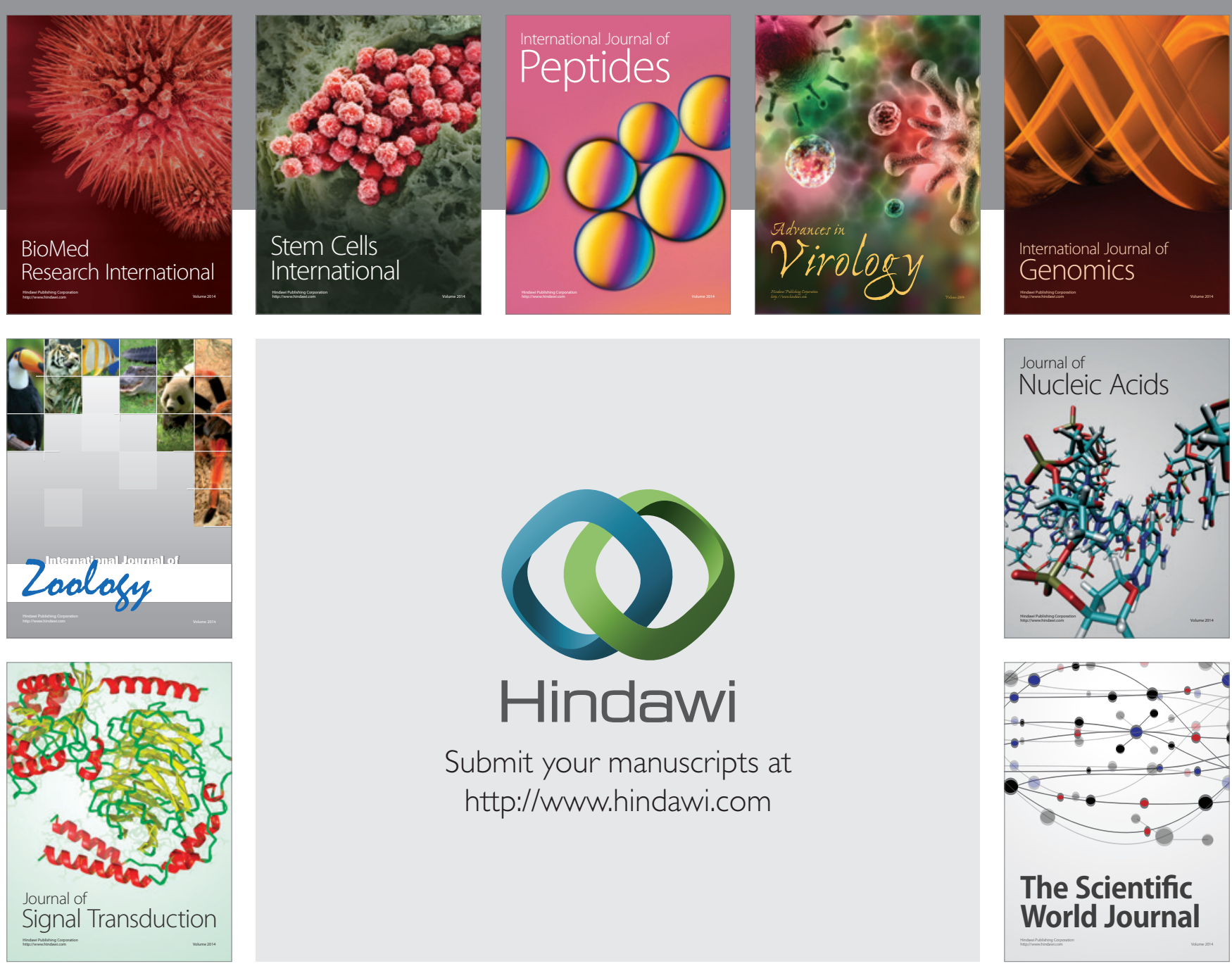

Submit your manuscripts at

http://www.hindawi.com
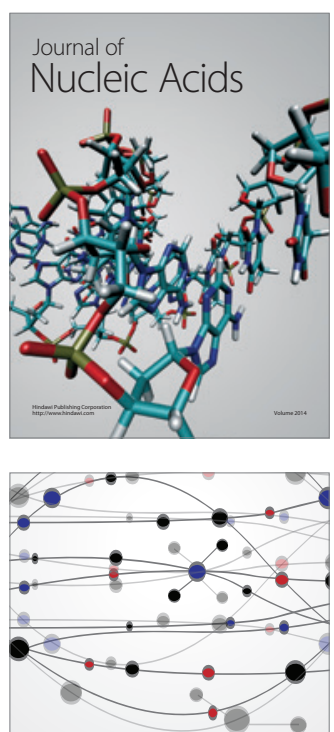

The Scientific World Journal
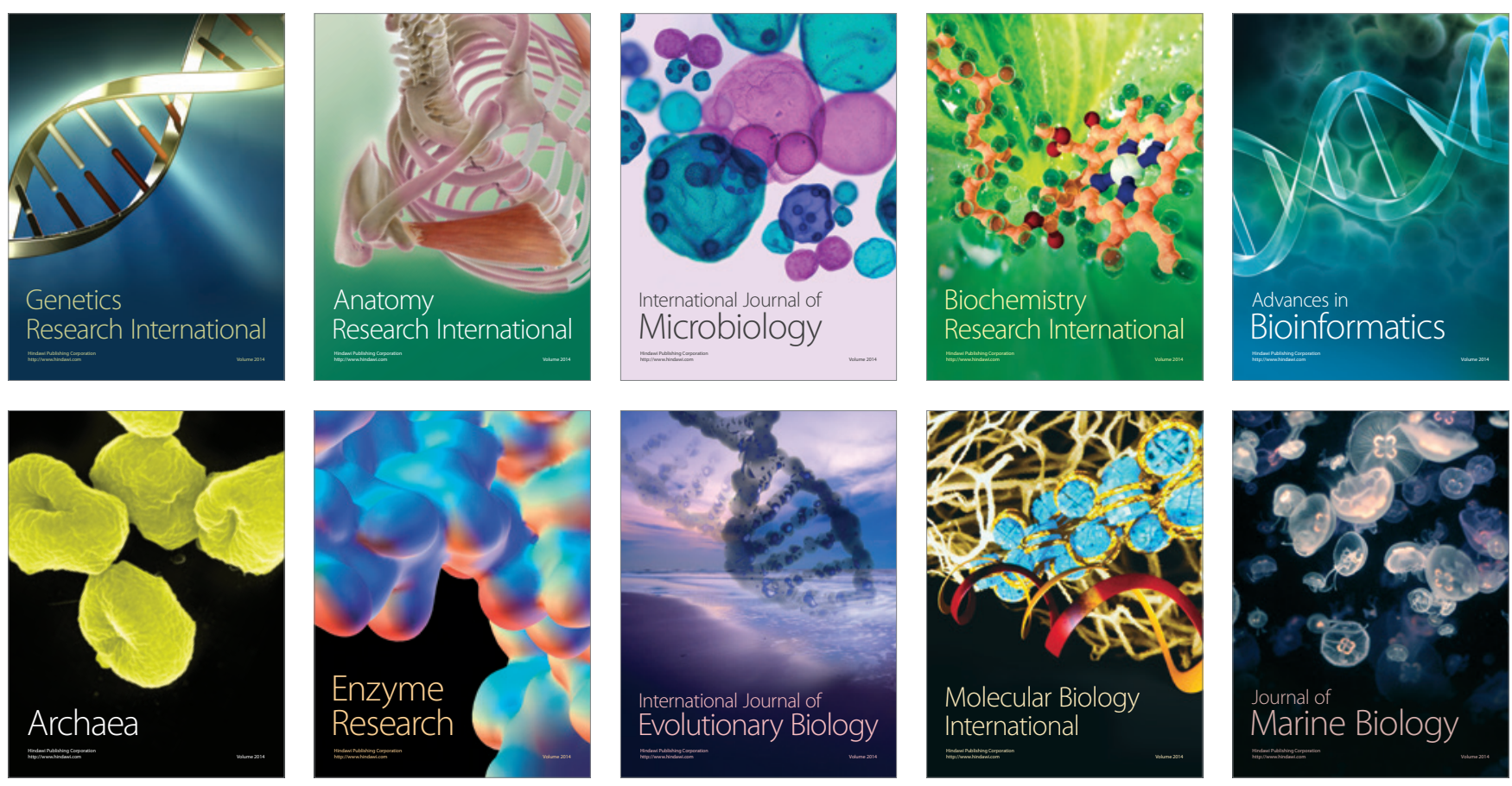